\title{
The Importance of Methyl-Branched Cuticular Hydrocarbons for Successful Host Recognition by the Larval Ectoparasitoid Holepyris sylvanidis
}

\author{
Sarah Awater-Salendo ${ }^{1,2} \cdot$ Hartwig Schulz $^{1} \cdot$ Monika Hilker $^{2} \cdot$ Benjamin Fürstenau $^{1}$ (D)
}

Received: 30 June 2020 / Revised: 29 September 2020 / Accepted: 13 October 2020 / Published online: 29 October 2020

(C) The Author(s) 2020

\begin{abstract}
Cuticular hydrocarbons (CHCs) of host insects are used by many parasitic wasps as contact kairomones for host location and recognition. As the chemical composition of $\mathrm{CHCs}$ varies from species to species, the $\mathrm{CHC}$ pattern represents a reliable indicator for parasitoids to discriminate host from non-host species. Holepyris sylvanidis is an ectoparasitoid of beetle larvae infesting stored products. Previous studies demonstrated that the larval CHC profile of the confused flour beetle, Tribolium confusum, comprises long chain linear and methyl-branched alkanes (methyl alkanes), which elicit trail following and host recognition in H. sylvanidis. Here we addressed the question, whether different behavioral responses of this parasitoid species to larvae of other beetle species are due to differences in the larval CHC pattern. Our study revealed that $H$. sylvanidis recognizes and accepts larvae of T. confusum, T. castaneum and T. destructor as hosts, whereas larvae of Oryzaephilus surinamensis were rejected. However, the latter species became attractive after applying a sample of $T$. confusum larval CHCs to solvent extracted larvae. Chemical analyses of the larval extracts revealed that CHC profiles of the Tribolium species were similar in their composition, while that of O. surinamensis differed qualitatively and quantitatively, i.e. methyl alkanes were present as minor components on the cuticle of all Tribolium larvae, but were absent in the $O$. surinamensis $\mathrm{CHC}$ profile. Furthermore, the parasitoid successfully recognized solvent extracted $T$. confusum larvae as hosts after they had been treated with a fraction of methyl alkanes. Our results show that methyl alkanes are needed for host recognition by H. sylvanidis.
\end{abstract}

Keywords Bethylidae $\cdot$ GC-MS analysis $\cdot$ Host recognition $\cdot$ Methyl alkanes $\cdot$ Stored product pest $\cdot$ Tenebrionidae $\cdot$ Tribolium

\section{Introduction}

Cuticular hydrocarbons (CHCs) are present on the surface of all insects and have several important functions, ranging from protection against desiccation to mediation of intra- and interspecific communication (Gibbs 1998; Ingleby 2015; Menzel et al. 2017; Otte et al. 2018). CHCs play a key role in the

Supplementary Information The online version of this article (https:// doi.org/10.1007/s10886-020-01227-w) contains supplementary material, which is available to authorized users.

Benjamin Fürstenau

benjamin.fuerstenau@julius-kuehn.de

1 Institute for Ecological Chemistry, Plant Analysis and Stored Product Protection, Julius Kühn Institute, Federal Research Centre for Cultivated Plants, Königin-Luise-Str. 19, 14195 Berlin, Germany

2 Dahlem Centre of Plant Science, Institute of Biology, Applied Zoology/Animal Ecology, Freie Universität Berlin, Haderslebener Str. 9, 12163 Berlin, Germany chemical communication of many insect species, serving as contact sex pheromones and kairomones for recognition of gender, caste, nest mates, mutualistic partners, and hosts (Howard and Blomquist 2005). The information conveyed by $\mathrm{CHCs}$ is based on various CHC types, which may be linear or methyl branched, saturated or unsaturated hydrocarbons with different chain lengths. The CHC patterns vary from species to species; some of these patterns are simple and contain only a few compounds, while others are composed of highly complex multi-component blends with up to $100 \mathrm{com}-$ pounds (Blomquist and Bagnères 2010).

Despite the complexity of insect $\mathrm{CHC}$ profiles, many studies pointed out that in general only a small number of components are involved in the intra- and interspecific communication of insects. For some species, single compounds of a specific CHC blend are sufficient to elicit behavioral responses (Colazza et al. 2007; Guédot et al. 2009; Kühbandner et al. 2012b; Rutledge et al. 2009), whereas for others a combination of several CHCs is required (Sakata et al. 2017; Spikes et al. 2010; Sugeno et al. 2006; Würf et al. 2020). A few 
studies have demonstrated the behavioral activity of straight chain (linear) alkanes and alkenes (Colazza et al. 2007; Dani et al. 2005; Ginzel et al. 2003b). In many cases, however, methyl alkanes have turned out to be the behavior inducing cues (e.g. Guédot et al. 2009; Ginzel et al. 2003a; Lacey et al. 2008; Ruther et al. 2011; Sakata et al. 2017; Silk et al. 2011).

Several species-specific CHCs have already been identified to be used by parasitic wasps to discriminate between mating partners or host species (Ablard et al. 2012; Kühbandner et al. $2012 b$ ). For generalist parasitoids, it is assumed that their foraging behavior is mediated by general cues released by different hosts (Steidle and Van Loon 2003), but only little is known about chemical cues used by parasitoid species that attack a broader range of host species.

The polyphagous, cosmopolitan ectoparasitoid Holepyris sylvanidis (Bréthes 1913) (Hymenoptera: Bethylidae) has been described to parasitize larvae of various beetle taxa (i.e. Cucujidae, Silvanidae, Tenebrionidae) infesting processed plant products (Amante et al. 2017b; Evans 1969; Hagstrum and Subramanyam 2009). Adults and larvae of these beetles are major pests of stored grain or wheat flour and occur worldwide in warehouses and in the food-processing industry (Hertlein et al. 2011; Vassilakos et al. 2012).

In a previous study we showed that $H$. sylvanidis responds to larval CHCs of its preferred host, the confused flour beetle Tribolium confusum (Du Val 1863) (Coleoptera: Tenebrionidae). The parasitoid uses the CHCs of the host larvae for host finding at short range by following CHC trails laid by larvae on the substrate (Fürstenau and Hilker 2017). The CHC profiles of some other potential host species of $H$. sylvanidis have also been chemically analyzed in the past (Baker et al. 1978; Howard et al. 1995; Lockey 1978), but their influence on host recognition or host selection of parasitoids has not yet been examined. Therefore, it is still unknown, whether $H$. sylvanidis recognizes suitable host larvae by a CHC pattern, which different host species have in common.

To address this question, we selected three beetle species which have been described as potential host species of H. sylvanidis; (i) T. confusum, (ii) the red flour beetle T. castaneum (Herbst 1797) (Coleoptera: Tenebrionidae) and (iii) the sawtoothed grain beetle, Oryzaephilus surinamensis (Linnaeus, 1758) (Coleoptera: Silvanidae). In addition to the aforementioned study by Fürstenau and Hilker (2017), several previous studies have shown that $T$. confusum and the closely related species $T$. castaneum are accepted as hosts by $H$. sylvanidis (Amante et al. 2017a, 2018; Ahmed et al. 1997; Fürstenau et al. 2016). Because of cooccurrence of $H$. sylvanidis and $O$. surinamensis in storage facilities (e.g. Eliopoulos et al. 2002) this grain beetle has been also considered a potential host species (Amante et al. 2017b; Hagstrum and Subramanyam 2009). However, it is unknown as yet, whether $H$. sylvanidis indeed accepts this species as host and whether the parasitoid can successfully develop on this species. Moreover, the destructive flour beetle, Tribolium destructor (Uyttenboogaart 1934) (Coleoptera: Tenebrionidae), a distantly related species of $T$. confusum and $T$. castaneum, was also included as a fourth species in the investigation without ever being described as a host of $H$. sylvanidis. However, it is interesting to note that adults of all three Tribolium spp. release 1-pentadecene, a volatile compound, which is considered to be part of the flour beetle aggregation pheromone (Arnaud et al. 2002; Verheggen et al. 2007) and has also been demonstrated to attract $H$. sylvanidis (Fürstenau et al. 2016).

The aim of the present study was to answer the following questions: (i) Do H. sylvanidis females recognize and accept larvae of different stored product pest beetle species as hosts? (ii) Does the larval $\mathrm{CHC}$ profile of a successfully recognized host species differ from that of a non-host? (iii) Which compounds of the $\mathrm{CHC}$ profile of a potential host species act as contact cues for successful host recognition by $H$. sylvanidis?

We analyzed hexane extracts of larvae of the aforementioned four beetle species by gas chromatography coupled with mass spectrometry (GC-MS) and compared the $\mathrm{CHC}$ profiles. In order to find out, whether methyl alkanes are responsible for host recognition by $H$. sylvanidis, we isolated them from larval extracts of $T$. confusum. Subsequently, the behavioral responses of $H$. sylvanidis to live and dead larvae of the four beetle species, to crude larval extracts of a host or a non-host species and to a fraction of methyl alkanes of the T. confusum CHC profile were tested in contact bioassays.

\section{Methods and Materials}

Insects The four stored-product pest beetles and potential host species of $H$. sylvanidis, $T$. castaneum, T. confusum, $T$. destructor, and O. surinamensis were reared in the laboratory of the Institute for Ecological Chemistry, Plant Analysis and Stored Product Protection (Julius Kühn Institute (JKI), Berlin, Germany). The host insects were reared in a climate chamber in permanent darkness at $25 \pm 1{ }^{\circ} \mathrm{C}$ and $65 \pm 5 \% \mathrm{RH}$.

The three analyzed Tribolium spp. were kept on the same feeding substrate, a mix of finely ground wheat grist (Triticum aestivum Linnaeus 1753) and wheat flour (Type 405, Kaufland, Neckarsulm, Germany, mix =1:1), in $400 \mathrm{ml}$ glass jars. Rearing of $T$. confusum was conducted as described by Fürstenau et al. (2016). The rearing protocol for T. castaneum and $T$. destructor was similar, but modified as follows: Two weeks after emergence from the pupal stage, 150 adults of $T$. castaneum and 50 adults of $T$. destructor were taken from the permanent rearing at the JKI and each placed in a glass jar filled with $150 \mathrm{ml}$ of feeding substrate. The adults (males and females) were left in the jars for one week and could mate and oviposit during this time. Thereafter, they were separated from the feeding substrate by sieving (mesh size $=710 \mu \mathrm{m}$ ) and 
transferred to another jar filled with fresh feeding substrate. The egg-infested substrate was stored for subsequent larval development. Approximately five weeks after oviposition, Tribolium larvae reached the 4th instar level, the (most) preferred host stage for $H$. sylvanidis. For our rearing, we used adult beetles for at maximum two weeks before replacing them by freshly emerged adults from the permanent rearing.

Oryzaephilus surinamensis was reared on coarsely ground wheat grist. Fifty adults (males and females) were kept in a $400 \mathrm{ml}$ glass jar. After mating and oviposition for one week, the adults were removed manually with forceps and placed in a jar filled with new feeding substrate. Beetles were used for at maximum three weeks before they were replaced by new (freshly emerged) ones for the further rearing. The substrate infested with $O$. surinamensis eggs was stored for subsequent larval development. In each jar we additionally placed a tissue paper to provide a good shelter for the latest instar larvae before pupation and to facilitate the removal of $O$. surinamensis larvae for following bioassays and chemical analysis. Larvae were removed approximately four weeks after oviposition when O. surinamensis larvae were 4th instars.

The parasitoid species $H$. sylvanidis was reared on T. confusum larvae in a climate chamber $\left(25 \pm 1{ }^{\circ} \mathrm{C}, 57 \pm 5 \%\right.$ RH). Previous studies had shown that experience with host or host-associated products (e.g. host exuviae or feces) just after emergence can induce behavioral changes in the parasitoid, resulting in reinforcement of inherited host preference (Barron 2001). Therefore, unexperienced ("naïve") H. sylvanidis females were used in the host recognition bioassays to exclude any biased effects.

To obtain naïve $H$. sylvanidis, one- to seven-day-old females were collected from the permanent rearing at the JKI (Berlin, Germany) and placed individually with one conspecific male in a Petri dish (94 mm diam., $16 \mathrm{~mm}$ height). Each Petri dish was provided with approx. $50 T$. confusum 4 th -5 th instars as hosts and $1.8 \mathrm{~g}$ ground wheat grist as food for the host larvae. A honey drop was applied onto the inner surface of the Petri dish lid for carbohydrate nutrition. After having located a host larva, the $H$. sylvanidis female paralyzes the host and pulls it to a sheltered site prior to laying its egg onto the host larva. Therefore, five pipette tips $(0.1-20 \mu \mathrm{l}$, Carl Roth, Karlsruhe, Germany) were offered in each Petri dish as hiding place for paralyzed host larvae. To prevent parasitoids from escaping, Petri dishes were sealed with parafilm. Tribolium confusum larvae were taken from our stock culture. The adult parasitoids could mate and oviposit onto the host larvae for one week. Thereafter, they were transferred to a new Petri dish to mate and oviposit for another week before they were replaced by newly emerged ones. Parasitized host larvae were separated from unparasitized ones and placed individually in a Petri dish $(5.0 \mathrm{~cm}$ diam.). After pupation of the parasitoid larvae (approx. one week after oviposition) we gently removed host exuviae and larval feces of $T$. confusum with a brush. Thus, freshly emerging $H$. sylvanidis adults had no chance to experience chemical cues of their rearing host, T. confusum. Naïve H. sylvanidis adult progenies emerged four to six weeks after oviposition and could feed on a drop of honey. Newly emerged parasitoids were separated by sex. Unmated, naïve, one- to four-day-old females were used in bioassays, while older females and males were discarded.

Preparation of Crude Larval Extracts from Potential Host Species For chemical analyses of the $\mathrm{CHC}$ profiles of potential host species and subsequent contact bioassays, we prepared the following larval extracts: (1) crude extracts from 4th instar larvae of each species ( $T$. castaneum, $T$. confusum, $T$. destructor and $O$. surinamensis) for GC-MS analysis, (2) crude extracts from 4th instar larvae of $T$. confusum and $O$. surinamensis for contact bioassays (dummy test I, treatments \#7,8,11,12 in Table 1), (3) crude extracts from $T$. confusum 4 th instar larvae for separation of $n$-alkanes from methyl branched ones and for contact bioassays testing the different CHC samples (dummy test II, treatment \#15-16 in Table 1).

A previous study by Fürstenau and Hilker (2017) demonstrated that the chemical composition of processed $\mathrm{CHC}$ extracts of $T$. confusum, which had been purified via solid-phase extraction, did not differ from crude extracts of the same beetle species. Therefore, we prepared and used only crude larval extracts of the aforementioned beetle species for our chemical analyses and bioassays.

(1) Crude larval extracts of the four potential host beetle species for chemical analysis were prepared by a procedure slightly modified from that described by Fürstenau and Hilker (2017). Preliminary tests revealed that only small amounts of compounds can be extracted from the cuticle of $O$. surinamensis larvae. Therefore, crude extracts of $O$. surinamensis larvae $(N=20)$ were prepared by immersing 90 larvae in $120 \mu \mathrm{l} n$-hexane (analytical purification $>98 \%$, VWR, Radnor, USA) for $10 \mathrm{~min}$ at ambient temperature. We concentrated the extract under a gentle stream of nitrogen and stored it at $-20{ }^{\circ} \mathrm{C}$ for further analysis. The 4th instar larvae of the studied host species differed somewhat in size and weight. For example, larvae of $O$. surinamensis were smaller (approx. 4 $5 \mathrm{~mm}$ ) than those of Tribolium spp. (approx. 6-10 mm). To prepare crude larval extracts with comparable larval biomass per solvent, we calculated the mean weight of 10 samples with 90 O. surinamensis larvae each (= $59.03 \mathrm{mg} \pm 0.66)$. We took this value to determine the number of larvae to be used for the other beetle species (T. confusum $=25$ larvae, $T$. castaneum $=28$ larvae, T. destructor $=21$ larvae). Thereafter, crude larval extracts of Tribolium spp. ( $N=20$ for each species) were prepared as described for those of $O$. surinamensis. To 
Table 1 Overview of contact bioassays for analyses of host recognition behavior by Holepyris sylvanidis females

\begin{tabular}{|c|c|c|c|c|c|}
\hline Bioassay & \# & Potential host species & State $^{\mathrm{a}}$ & Treatment $^{\mathrm{b}}$ & $N^{c}$ \\
\hline \multirow{4}{*}{$\begin{array}{l}\text { A) Host recognition - } \\
\text { Influence of beetle species }\end{array}$} & 1 & T. castaneum & $\mathrm{L} / \mathrm{D}$ & untreated & 36 \\
\hline & 2 & T. confusum & $\mathrm{L} / \mathrm{D}$ & untreated & 36 \\
\hline & 3 & T. destructor & $\mathrm{L} / \mathrm{D}$ & untreated & 36 \\
\hline & 4 & O. surinamensis & $\mathrm{L} / \mathrm{D}$ & untreated & 36 \\
\hline \multirow{8}{*}{$\begin{array}{l}\text { B) Dummy test I- } \\
\text { Influence of host larval CHCs }\end{array}$} & 5 & T. confusum & $\mathrm{D}$ & untreated & 30 \\
\hline & 6 & “ & $\mathrm{D}$ & extracted $^{\dagger}$ & 30 \\
\hline & 7 & “ & $\mathrm{D}$ & extracted $^{\dagger}+$ re-applied $O$. sur-larval extract & 30 \\
\hline & 8 & “ & $\mathrm{D}$ & extracted $^{\dagger}+$ re-applied $T$. con-larval extract ${ }^{\ddagger}$ & 30 \\
\hline & 9 & O. surinamensis & $\mathrm{D}$ & untreated & 30 \\
\hline & 10 & “ & $\mathrm{D}$ & extracted ${ }^{\dagger}$ & 30 \\
\hline & 11 & “ & $\mathrm{D}$ & extracted $^{\dagger}+$ re-applied $O$. sur-larval extract ${ }^{*}$ & 30 \\
\hline & 12 & “ & $\mathrm{D}$ & extracted $^{\dagger}+$ re-applied $T$. con-larval extract ${ }^{*}$ & 30 \\
\hline \multirow{4}{*}{$\begin{array}{l}\text { C) Dummy test II- } \\
\text { Influence of host larval } \\
\text { CHC fractions }\end{array}$} & 13 & T. confusum & $\mathrm{D}$ & untreated & 40 \\
\hline & 14 & “ & $\mathrm{D}$ & extracted $^{\dagger}$ & 40 \\
\hline & 15 & “ & $\mathrm{D}$ & $\begin{array}{l}\text { extracted }^{\dagger}+\text { re-applied } T \text {. con-larval extract } \\
\quad \text { - complete CHC profile }\end{array}$ & 40 \\
\hline & 16 & “ & $\mathrm{D}$ & $\begin{array}{l}\text { extracted }^{\dagger}+\text { re-applied methyl-branched } \\
\text { CHC-fraction }\end{array}$ & 40 \\
\hline
\end{tabular}

${ }^{\text {a }}$ Live $(\mathbf{L})$ or differntly treated dead (D) larvae were offered to parasitoids. Larvae were killed at $-20{ }^{\circ} \mathrm{C}$. Behavioral responses and searching time until successful host recognition were recorded for a period of $300 \mathrm{~s}$

${ }^{\mathrm{b}}$ Different treatments for dead larvae as test stimuli:

${ }^{\dagger}$ To remove cuticular hydrocarbons, larvae were extracted for 10 min with $n$-hexane

$\$ 2 \mu \mathrm{l}$ (= $1 / 2$ larval equivalent, LE) of $T$. confusum (T. con) or O. surinamensis (O. sur) crude larval extract were re-applied to extracted larvae

* $1 \mu 1$ (= $1 / 2 \mathrm{LE})$ of $T$. con or $O$. sur crude larval extracts were re-applied to extracted larvae

${ }^{\wedge} 2 \mu \mathrm{l}(=1 / 2 \mathrm{LE})$ of $T$. con larval extract were re-applied to extracted larvae before fractionation with $5 \AA$ Åmolecular sieves

${ }^{\wedge} 2 \mu 1(=1 / 2 \mathrm{LE})$ of methyl alkane fraction of $T$. con larval extract were re-applied to extracted larvae after fractionation with $5 \AA$-molecular sieves

${ }^{\mathrm{c}}$ Number of replicates

quantify the amount of host larval CHCs, extracts were re-dissolved in $50 \mu 1 n$-hexane containing 1-eicosene as internal standard (IS, $10.4 \mathrm{ng} \mu \mathrm{l}^{-1}$, Sigma-Aldrich, Taufkirchen, Germany).

(2) Crude larval extracts for contact bioassays were produced as follows: Ten larvae of T. confusum or of $O$. surinamensis were immersed in $100 \mu \mathrm{l} n$-hexane and removed after $10 \mathrm{~min}$ before the supernatant was dried under a gentle stream of nitrogen. For the re-application of CHCs on dead and extracted T. confusum larvae, the extracted CHCs were re-dissolved in $40 \mu \mathrm{l} n$-hexane (treatments \#7-8 in Table 1). For re-application of CHCs on dead and extracted $O$. surinamensis larvae, the extracted CHCs were re-dissolved in $20 \mu \mathrm{l} n$-hexane (treatments \#11-12 in Table 1). Since T. confusum larvae are larger and thicker than those of $O$. surinamensis, $2 \mu \mathrm{l}$ of crude larval extract were required to uniformly impregnate a $T$. confusum larva. In contrast, applying $1 \mu \mathrm{l}$ was enough to evenly cover an $O$. surinamensis larva.
(3) Crude larval extracts of $T$. confusum for fractionation and further contact bioassays with different $\mathrm{CHC}$ samples were produced by following a method described by Bello et al. (2015). Approximately 2000 freshly killed $T$. confusum larvae were extracted in $5 \mathrm{ml} n$-hexane for $10 \mathrm{~min}$. The supernatant was concentrated to ca. $1000 \mu \mathrm{l}$ under a gentle stream of nitrogen. To purify the alkanes (both linear and methyl branched), we loaded the crude extract onto an isolute silica gel column (100 mg, Biotage, Uppsala, Sweden), which had been pre-conditioned by rinsing two times with $1 \mathrm{ml}$ dichloromethane (>99\%, Merck, Darmstadt, Germany) and $1 \mathrm{ml} n$-hexane. The sample was eluted from the column by applying four times $1 \mathrm{ml} n$-hexane.

From this eluate (purified CHCs), we took (i) $450 \mu 1(\approx 200$ LE of $T$. confusum) for subsequent contact bioassays and (ii) $100 \mu \mathrm{l}(\approx 44 \mathrm{LE}$ of $T$. confusum) for chemical analysis by GC-MS; both types of samples were stored at $-20{ }^{\circ} \mathrm{C}$. 
To separate the methyl alkanes from linear ones, the remaining eluate was transferred to a $25 \mathrm{ml}$ vial and concentrated to dryness before being re-dissolved in $5 \mathrm{ml}$ isooctane (Merck, Darmstadt, Germany). We added $100 \mathrm{mg}$ of activated $5 \AA$-molecular sieves (SigmaAldrich, Taufkirchen, Germany) per mg of sample, while the vial was flushed with nitrogen for $5 \mathrm{~min}$. To activate the molecular sieves, they had previously been dried in a muffle furnace at $300{ }^{\circ} \mathrm{C}$ for $15 \mathrm{~h}$. In the airtight-sealed vial, the extract was then magnetically stirred at ambient temperature for $18 \mathrm{~h}$. Thereafter, the supernatant containing isolated methyl alkanes was removed and filtered through a Whatman filter paper $(9.0 \mathrm{~cm}$ diam.). Prior to storage of this supernatant for later contact bioassays, $100 \mu \mathrm{l}(\approx 44 \mathrm{LE}$ of $T$. confusum) were taken for GC-MS analysis. In total, we fractionated three samples of larval extracts $(N=3)$. For chemical analysis, we used $100 \mu \mathrm{l}$ each of the supernatant containing isolated methyl alkanes and of the eluate containing purified CHCs $(N=3$ per sample type). Under a gentle stream of nitrogen, we concentrated each sample to dryness before adding $50 \mu \mathrm{l}$ $n$-hexane containing 1-eicosene (10.4 ng $\left.\mu^{-1}\right)$ as IS.

To test the influence of different structural groups of CHCs on the host recognition behavior of $H$. sylvanidis, we used purified CHCs (mixture of linear and methyl alkanes) as well as isolated methyl alkanes, which were obtained by separation from $T$. confusum crude larval extracts. For treatment \#15 (Table 1), we concentrated $46 \mu \mathrm{l}$ of each sample with purified CHCs $(\approx 20$ LE of T. confusum) under a gentle stream of nitrogen and dissolved each sample in $80 \mu \mathrm{l} n$-hexane. For treatment \#16 (Table 1$)$, we took $50 \mu \mathrm{l}(\approx 20$ LE of $T$. confusum) of each sample with isolated methyl alkanes and prepared the extracts as described for those in treatment \#15.

\section{GC-MS Analysis of Crude Larval Extracts of Potential Host} Species and Different CHC Samples of T. confusum Larvae GC-MS analyses of crude larval extracts and different $\mathrm{CHC}$ samples of $T$. confusum larval extracts were performed on a GCT Premier - TOF Mass Spectrometer (Waters, Milford, USA) coupled to a GC System 7890A (Agilent Technologies, Waldbronn, Germany). One $\mu$ l of each sample was injected in splitless mode, keeping the injector at $250{ }^{\circ} \mathrm{C}$ with helium as carrier gas $\left(1 \mathrm{ml} \mathrm{min}{ }^{-1}\right)$. The oven temperature program started at $40{ }^{\circ} \mathrm{C}$, which was held for $4 \mathrm{~min}$ and increased then at $10^{\circ} \mathrm{C} \min ^{-1}$ to $300{ }^{\circ} \mathrm{C}$. The final temperature was held for $20 \mathrm{~min}$. Samples were separated on a $30 \mathrm{~m} \mathrm{HP}$ $5 \mathrm{MS}$ capillary column $(250 \mu \mathrm{m}$ diam., $0.25 \mu \mathrm{m}$ film thickness, Agilent JandW Scientific). After a solvent delay of $5 \mathrm{~min}$, masses were scanned every $0.9 \mathrm{~s}$ with a range from 50 to $600 \mathrm{~m} / \mathrm{z}$ (electronic impact [EI] ionization $=70 \mathrm{eV}$, source temperature $=230^{\circ} \mathrm{C}$ ).
For structure assignments of detected compounds, an authentic $n$-alkane standard (n-C7-n-C40, Sigma-Aldrich, Taufkirchen, Germany) was additionally injected. Linear alkanes were identified by comparing their mass spectra and the calculated retention indices (RIs) with those of the $n$-alkane standard. In contrast, no reference compounds of the methyl alkanes were available to us. For each compound, we tentatively determined the position of its methyl branching based on the characteristic mass spectrometric fragmentation and the calculated RIs according to van den Dool and Kratz (1963). We further compared the RI and the fragmentation pattern with those published by Lockey (1978), Hebanowska et al. (1989, 1990), Howard et al. (1995), Spiewok et al. (2006), Geiselhardt et al. (2009), Svensson et al. (2014), Gerhardt et al. (2016), and Fürstenau and Hilker (2017).

Individual compounds were quantified relative to the peak area of the IS. CHC samples were standardized by calculating the mean amount of each compound (in ng) per one larval equivalent (LE).

\section{Contact Bioassay: Host Finding and Recognition Behavior To} analyze the response of one-to four-day-old, naïve H. sylvanidis females to 4th instar (live and dead) larvae of (i) T. confusum, (ii) T. castaneum, (iii) T. destructor and (iv) $O$. surinamensis, we performed a series of different contact bioassays (Table 1). The bioassay methods were similar to those described by Fürstenau and Hilker (2017). The parasitoid behavior was observed in a test arena, which consisted of a circle $(9.0 \mathrm{~cm}$ diam.) drawn on a sheet of white paper and covered by the lid of a plastic Petri dish $(94 \mathrm{~mm}$ diam., $16 \mathrm{~mm}$ height). To avoid any external interference, the test arena was surrounded by a cardboard box $(70.5 \mathrm{~cm} \mathrm{x} 44.5 \mathrm{~cm}$ x $41 \mathrm{~cm})$. For an even illumination, a strip of light-emitting diodes $(\lambda=625 \mathrm{~nm}$, Barthelme $\mathrm{GmbH} \& \mathrm{Co}$, Nürnberg, Germany) was located $5 \mathrm{~cm}$ above the box. All bioassays took place at $25 \pm 1{ }^{\circ} \mathrm{C}$. Live or differently treated dead larvae of each species were placed individually in the center of the test arena. Beetle larvae were killed by freezing at $-20{ }^{\circ} \mathrm{C}$ for $2 \mathrm{~h}$ and allowed to warm to room temperature for $30 \mathrm{~min}$ prior to biotest start. In three different experimental set ups we tested the influence of the following host stimuli on the host recognition behavior of $H$. sylvanidis (Table 1):

A) untreated larvae of the previously mentioned beetle species

B) crude larval extracts (CHC profiles) of $T$. confusum or O. surinamensis, respectively, applied onto extracted larvae of these two species

C) different $\mathrm{CHC}$ samples of $T$. confusum larvae (including one sample of isolated methyl alkanes) applied onto extracted $T$. confusum larvae

Untreated as well as extracted larvae of the respective species were used as control treatments in bioassays B) and C). 
All bioassays were prepared by placing the host stimulus (a single live or dead larva) in the center of the test arena. When a crude larval host extract was applied onto a test larva, the solvent could evaporate for $1 \mathrm{~min}$ prior to release of the parasitoid into the arena. A bioassay began by releasing a single $H$. sylvanidis female onto the circle drawn on the sheet of paper lining the arena. The release side of parasitoids rotated clockwise to avoid any biased results due to possible side preference. Each individual parasitoid was observed for max. 300 s. As described by Fürstenau and Hilker (2017), we recorded (i) whether the parasitoid located a host larva and showed host recognition behavior when encountering it and (ii) determined the searching time until successful host recognition. We defined successful host recognition as the moment when $H$. sylvanidis bends its abdomen around the larva to start paralyzing it. Once the parasitoid successfully found and recognized its host, the experiment was stopped. Test individuals, host stimuli, and the paper lining the arena were replaced by new ones after each run. The lid of the Petri dish was cleaned with a $70 \%$ ethanol solution (>96\%, Berkel AHK, Ludwigshafen, Germany). When a parasitoid rested more than $50 \%$ of the observation time - less than 5\% (34 occasions) of all bioassays listed in Table 1 - the individual was not included in the statistical analysis and replaced by a new one, which showed active searching behavior. When live or dead larvae of Tribolium spp. and O. surinamensis were offered as potential hosts (bioassay "A"), each treatment was replicated 36 times $(N=36)$. When we studied the influence of crude larval extracts of $T$. confusum or $O$. surinamensis on host recognition of $H$. sylvanidis (bioassay "B"), we repeated each treatment 30 times $(N=30)$. When the influence of different CHC samples of $T$. confusum larvae on host recognition of $H$. sylvanidis were tested (bioassay "C"), each treatment was replicated 40 times $(N=40)$.

Bioassay: Host Acceptance and Oviposition To figure out whether the parasitoid accepts larvae of Tribolium spp. and O. surinamensis for oviposition, we conducted a further bioassay, which additionally allowed us to check the development of the parasitoid's offspring on the beetle larvae. Holepyris sylvanidis deposits a single egg onto a host larva and only a single parasitoid larva can develop per host (Amante et al. 2017a).

A naïve, one-to four-day-old $H$. sylvanidis was offered one live 4th instar larva in a Petri dish $(5.0 \mathrm{~cm}$ diam.) for a period of $24 \mathrm{~h}$. The potential host larva was provided with $0.4 \mathrm{~g}$ finely ground wheat grist. Furthermore, one pipette tip (0.1-20 $\mu$, Carl Roth, Karlsruhe, Germany) was offered as hiding place to the parasitoid since $H$. sylvanidis pulls paralyzed host larvae to a shelter site prior to oviposition. For each potential host species, we tested 40 female parasitoids on 40 host larvae $(N=40$ per species). After the 24-hour-exposure time to the parasitoid, the parasitized larvae were transferred to a climate chamber $\left(25 \pm 1{ }^{\circ} \mathrm{C}, 57 \pm 5 \% \mathrm{RH}\right.$, permanent darkness) for further development. Larvae onto which the parasitoid had oviposited, were recognized by the parasitoid's egg on the cuticle of the host larval. Unparasitized larvae were classified as notaccepted hosts. After four weeks, the number of emerging parasitoids per host species were counted (= successful host acceptance). Host larvae, which were not accepted as hosts and those on which $H$. sylvanidis larvae had not completed its development, were counted as failed host acceptance.

Statistical Analysis All statistical analysis were computed in "R", version 3.6.1 (R Core Team 2019), except of the SIMPER analysis, which was performed in "PAST", version 3.26 (Hammer et al. 2001).

For an across-beetle-species-comparison, we prepared data sets obtained by chemical analyses of the $\mathrm{CHC}$ profiles as follows. For some peaks, the mass spectrum and RI indicated that several internally branched alkanes (branching at position $10,11,12,13,14$ or 15) co-eluted. The RIs of these compounds differed slightly among the Tribolium species due to the different positions of the methyl branching. Therefore, we pooled these internally branched alkanes. Additionally, we only included compounds, which were present in more than $50 \%$ of all extracts of a beetle species. When one of the selected compounds was below the detection limit in some extracts of a beetle species, we handled the missing compound as follows. To avoid any bias in the subsequent statistical analysis, we generated a random peak for each missing value by the "rnorm()"-function in "R". Since the missing compound had been detected in other extracts of a beetle species, we selected the smallest peak area, which this compound had in these extracts, as mean and calculated the standard deviation based on the four smallest peak areas. In total, 43 pseudo peaks were generated. Finally, we normalized all selected compounds by calculating the quantitative contribution of each compound to one LE of the beetle species.

To statistically compare the $\mathrm{CHC}$ patterns of the tested beetle species, we calculated relative amounts of detected compounds in $1 \mathrm{LE}$ of each beetle species and summed up all amounts to $100 \%$. Based on the Bray-Curtis dissimilarity, a one-way analysis of similarity (ANOSIM) was then performed with 99,999 random permutations using the package "vegan" (version 2.5-6, Oksanen et al. 2019) in "R". The dissimilarity of groups is stated by the $R$-value, ranging between 0 and 1 . An $R$-value close to 1 indicates a clear discrimination, whereas an $R$-value close to 0 indicates a high similarity (Clarke 1993). We also applied an analysis of similarity percentages (SIMPER) to identify compounds, which contribute the most (i) to the dissimilarity between the $\mathrm{CHC}$ profiles of Tribolium spp. or (ii) to the dissimilarity between the $\mathrm{CHC}$ profiles of Tribolium spp. and $O$. surinamensis. The differences among $\mathrm{CHC}$ profiles were visualized by performing a non-metric multidimensional scaling (NMDS) calculated on Bray-Curtis dissimilarity. The stress value associated with 
NMDS indicates how close the algorithm of NMDS fits to the used data set. A NDMS with a stress value $<0.1$ indicates a good fit of the NMDS ordination or low data distortion (Clarke 1993; Dexter et al. 2018).

A comparison of the behavioral responses of $H$. sylvanidis to the offered different host stimuli was based on (i) the host recognition rate per potential host species/treatment and (ii) the mean searching time until successful host recognition of the offered larva. The host recognition rate was analysed by the test of equality of proportions followed by a pairwise comparison of proportions with Bonferroni-Holm correction (Newcombe 1998a, b). When the parasitoid's response to dead, differently treated $O$. surinamensis larvae (treatments \#9-12 in Table 1) was tested in contact bioassays, results were evaluated by Fisher's exact test, followed by a pairwise comparison of proportions with Bonferroni-Holm correction. Since the Shapiro-Wilk test of normality revealed that the mean searching times required by the parasitoids were not normally distributed in all treatments, we applied the Kruskal-Wallis test for comparing the mean searching time of parasitoids exposed to different host stimuli. Thereafter, differences in the mean searching time between the different treatments were pairwise compared using Wilcoxon rank sum test with Bonferroni-Holm correction.

To analyze the host acceptance behavior of $H$. sylvanidis, we determined for each species the number of larvae successfully accepted as hosts and of those that were not. We recorded "successful host acceptance", when parasitoid offspring emerged from the host and "failed host acceptance", when host larvae were left unparasitized or when parasitoid larvae could not successfully develop inside the host. Finally, the proportions of successful and failed host acceptance were analyzed by the test of equality of proportions followed by a Bonferroni-Holm corrected pairwise comparison of proportions across the beetle species.

\section{Results}

\section{Comparison of Larval CHC Profiles of Possible Host Species} Our GC-MS analysis revealed that crude larval extracts of O. surinamensis and Tribolium spp. exclusively consist of saturated cuticular hydrocarbons (CHCs) with chain lengths from $n$-C25 to $n$-C 36 . In total, we identified 31 compounds, including $12 n$-alkanes, 14 monomethyl alkanes and five dimethyl alkanes (Table 2; Fig. 1a, b). The CHC profiles showed quantitative and qualitative differences between beetle species, but a common pattern within the genus Tribolium was observed (Table 2).

In Tribolium spp., a series of $n$-alkanes with chain lengths from $n$-C25 to $n$-C 33 represented the dominating group of substance in quantities of $93 \%$ (T. confusum), $90 \%$ (T. castaneum), and $79 \%$ (T. destructor). Most prominent components were $n$ -
C27 (T. confusum and T. destructor, entry 10) and $n$-C29 (T. castaneum, entry 22). $n$-Alkanes with more than 30 carbon atoms (entries 25, 26, 27) accounted for less than $10 \%$ of all $\mathrm{CHC}$ profiles analyzed from larvae of this genus. In addition to the fraction of $n$-alkanes, various monomethyl- and dimethyl alkanes were identified in larval extracts of the Tribolium spp. Based on the position of the methyl branch, monomethyl alkanes were either internally branched (branching at positions $10,11,12,13,14,15$ - since positional isomers could not be separated, co-occurrence is indicated by a slash) or externally branched (branching at position 3, 4 or 5), but both groups were present in all Tribolium extracts. Dimethyl alkanes were most abundant in the extracts of $T$. destructor (entries $5,15,17,21$ ), while only one dimethyl alkane was present in $T$. confusum (entry 15) and T. castaneum (entry 13). The mass spectra of two dimethyl alkanes (5,X-DiMeC27 and 3,X-DiMeC28; entries 15 and 17) did not allow a clear determination of the position of the second methyl group. Therefore, the unknown position was labelled by ' $\mathrm{X}$ ' (Table 2). Overall, seven methyl alkanes were found in all extracts of Tribolium spp. (3-MeC25, 11-/13-MeC27, 3-MeC27 and 12-/13-/14-MeC28; entries 4, 11, 14 and 18) (Table 2). Among these compounds, $11-\mathrm{MeC} 27$ and $13-\mathrm{MeC} 27$ were most abundant (T. confusum $=3 \%$, T. castaneum $=6 \%$, T. destructor $=7 \%$ ).

In contrast to the CHC profiles of Tribolium spp., the profile of $O$. surinamensis was less diverse and contained only linear alkanes, but no methyl alkanes. In total, the assigned peaks represented $n$-alkanes with chain lengths from $n$-C25 to $n$-C36 (Table 2; Fig. 1a). The most abundant component was $n$-C31 (entry 26), and compounds with more than 29 carbon atoms contributed to $77 \%$ of the total peak area (Table 2).

Comparison of the CHC profiles of Tribolium spp. larvae among each other and with those of $O$. surinamensis larvae by an ANOSIM showed a significant dissimilarity among the beetle species $(R=0.9988, P<0.001)$, which were clearly separated in the NDMS plot (Fig. 2). Tribolium spp., however, clustered closer together, indicating that the composition of their CHC profiles was more similar compared to O. surinamensis. This was confirmed by the overall average dissimilarity index calculated in SIMPER (Table S1); this index was smaller when comparing the Tribolium spp. among each other ( $T$. castaneum vs. $T$. confusum vs. $T$. destructor $=$ 38.96) than in the pairwise comparison between each Tribolium spp. and $O$. surinamensis (T. castaneum vs. $O$. surinamensis $=68.75, T$. confusum vs. $O$. surinamensis $=$ $68.58, T$. destructor vs. O. surinamensis $=79.79$, Table S2, S3, S4). In addition, the SIMPER analysis revealed that all compounds, which accounted most for the dissimilarity between Tribolium spp. as well as between each Tribolium spp. and $O$. surinamensis, were $n$-alkanes (>10\%). When considering the within-genus comparison of Tribolium spp., $n$-C25 (entry 1), $n$-C27 (entry 10), and $n$-C29 (entry 22) contributed together by $73 \%$ to the observed dissimilarity (Table S1). 
Table 2 Cuticular hydrocarbons identified from crude larval extracts of Tribolium confusum, T. castaneum, T. destructor and Oryzaephilus surinamensis

\begin{tabular}{|c|c|c|c|c|c|c|c|c|c|c|c|}
\hline \multirow[b]{2}{*}{ No. ${ }^{a}$} & \multirow[b]{2}{*}{ Compound $^{\mathrm{b}}$} & \multirow[b]{2}{*}{$\mathrm{RI}_{\mathrm{cal}}^{\mathrm{c}}$} & \multirow[b]{2}{*}{$\mathrm{RI}_{\text {lit }}{ }^{\mathrm{d}}$} & \multicolumn{2}{|l|}{ T. confusum ${ }^{\mathrm{e}}$} & \multicolumn{2}{|l|}{ T. castaneum $^{\mathrm{e}}$} & \multicolumn{2}{|l|}{ T. destructor ${ }^{\mathrm{e}}$} & \multicolumn{2}{|l|}{ O. surinamensis ${ }^{\mathrm{e}}$} \\
\hline & & & & Mean \pm SE (ng) & $(\%)$ & Mean \pm SE (ng) & $(\%)$ & Mean \pm SE (ng) & $(\%)$ & Mean \pm SE (ng) & $(\%)$ \\
\hline 1 & $n-\mathrm{C} 25$ & 2498 & 2500 & $3.35 \pm 0.39$ & 4.40 & $2.15 \pm 0.20$ & 3.11 & $16.76 \pm 2.11$ & 24.04 & $0.07 \pm 0.02$ & 1.90 \\
\hline 2 & 11-/13-MeC25 & 2533 & 2534 & & & $0.05 \pm 0.00$ & 0.07 & $0.08 \pm 0.01$ & 0.11 & & \\
\hline 3 & $5-\mathrm{MeC} 25$ & 2548 & 2550 & & & & & $0.36 \pm 0.04$ & 0.55 & & \\
\hline 4 & 3-MeC25 & 2572 & 2571 & $0.04 \pm 0.00$ & 0.05 & $0.27 \pm 0.03$ & 0.38 & $0.43 \pm 0.05$ & 0.64 & & \\
\hline 5 & 5,11-DiMeC25 & 2581 & 2577 & & & & & $0.32 \pm 0.03$ & 0.50 & & \\
\hline 6 & $n-\mathrm{C} 26$ & 2597 & 2600 & $0.67 \pm 0.05$ & 0.87 & $0.53 \pm 0.03$ & 0.78 & $1.89 \pm 0.15$ & 2.83 & $0.02 \pm 0.00$ & 0.65 \\
\hline \multirow[t]{2}{*}{7} & 10-/11-/12-MeC26 & 2634 & 2632 & $0.09 \pm 0.01$ & 0.11 & $0.10 \pm 0.01$ & 0.15 & & & & \\
\hline & 12-/13-MeC26 & & & & & & & $0.32 \pm 0.03$ & 0.48 & & \\
\hline 8 & 4-MeC26 & 2656 & 2656 & $0.03 \pm 0.00$ & 0.03 & & & $0.13 \pm 0.01$ & 0.20 & & \\
\hline 9 & 3-MeC26 & 2674 & 2673 & & & $0.13 \pm 0.02$ & 0.18 & $0.25 \pm 0.03$ & 0.37 & & \\
\hline 10 & $n-\mathrm{C} 27$ & 2700 & 2700 & $40.37 \pm 4.36$ & 49.55 & $19.13 \pm 0.95$ & 28.00 & $25.06 \pm 1.53$ & 38.63 & $0.16 \pm 0.02$ & 4.51 \\
\hline 11 & 11-/13-MeC27 & 2731 & 2731 & $2.28 \pm 0.18$ & 2.98 & $4.32 \pm 0.29$ & 6.29 & $4.75 \pm 0.33$ & 7.30 & & \\
\hline 12 & 5-MeC27 & 2748 & 2750 & $0.58 \pm 0.06$ & 0.72 & & & $0.80 \pm 0.06$ & 1.22 & & \\
\hline 13 & 11,15-DiMeC27 & 2758 & 2762 & & & $0.35 \pm 0.03$ & 0.51 & & & & \\
\hline 14 & 3-MeC27 & 2771 & 2773 & $1.16 \pm 0.13$ & 1.39 & $1.04 \pm 0.07$ & 1.52 & $1.52 \pm 0.11$ & 2.28 & & \\
\hline 15 & 5, X-DiMeC27 & 2778 & 2781 & $0.35 \pm 0.04$ & 0.43 & & & $1.95 \pm 0.13$ & 3.01 & & \\
\hline 16 & $n-\mathrm{C} 28$ & 2797 & 2800 & $5.84 \pm 0.68$ & 7.04 & $3.24 \pm 0.19$ & 4.71 & $1.78 \pm 0.11$ & 2.87 & $0.13 \pm 0.01$ & 3.46 \\
\hline 17 & 3,X-DiMeC28 & 2802 & 2807 & & & & & $2.15 \pm 0.12$ & 3.37 & & \\
\hline 18 & 12-/13-/14-MeC28 & 2830 & 2831 & $0.21 \pm 0.02$ & 0.26 & $0.20 \pm 0.02$ & 0.29 & $0.33 \pm 0.02$ & 0.51 & & \\
\hline 19 & 4-MeC28 & 2855 & 2856 & $0.09 \pm 0.01$ & 0.11 & $0.03 \pm 0.00$ & 0.05 & & & & \\
\hline 20 & 3-MeC28 & 2874 & 2872 & & & $0.00 \pm 0.00$ & 0.00 & & & & \\
\hline 21 & 4,14-DiMeC28 & 2886 & 2893 & & & & & $0.10 \pm 0.01$ & 0.16 & & \\
\hline 22 & $n-\mathrm{C} 29$ & 2899 & 2904 & $19.92 \pm 2.96$ & 22.71 & $30.36 \pm 1.67$ & 44.29 & $1.66 \pm 0.08$ & 2.73 & $0.45 \pm 0.03$ & 12.50 \\
\hline \multirow[t]{3}{*}{23} & 11-/13-MeC29 & 2928 & 2931 & $0.33 \pm 0.03$ & 0.41 & & & & & & \\
\hline & 11-/13-/15-МeC29 & & & & & $0.35 \pm 0.03$ & 0.51 & & & & \\
\hline & 11-/15-МeC29 & & & & & & & $0.18 \pm 0.01$ & 0.28 & & \\
\hline 24 & 3-MeC29 & 2970 & 2978 & $0.22 \pm 0.03$ & 0.26 & $0.33 \pm 0.02$ & 0.47 & & & & \\
\hline 25 & $n-\mathrm{C} 30$ & 2995 & 3000 & $1.33 \pm 0.13$ & 2.01 & $1.51 \pm 0.11$ & 2.22 & $1.22 \pm 0.11$ & 2.05 & $0.48 \pm 0.05$ & 12.55 \\
\hline 26 & $n-\mathrm{C} 31$ & 3094 & 3100 & $2.02 \pm 0.17$ & 2.85 & $2.26 \pm 0.19$ & 3.29 & $1.50 \pm 0.14$ & 2.52 & $0.97 \pm 0.08$ & 26.04 \\
\hline 27 & $n-\mathrm{C} 32$ & 3194 & 3200 & $1.19 \pm 0.15$ & 1.85 & $1.23 \pm 0.13$ & 1.80 & $1.23 \pm 0.12$ & 2.08 & $0.60 \pm 0.08$ & 14.60 \\
\hline 28 & $n-\mathrm{C} 33$ & 3295 & 3300 & $1.46 \pm 0.16$ & 1.96 & $0.97 \pm 0.10$ & 1.40 & $0.76 \pm 0.08$ & 1.27 & $0.49 \pm 0.08$ & 11.66 \\
\hline 29 & $n-\mathrm{C} 34$ & 3395 & 3400 & & & & & & & $0.29 \pm 0.06$ & 6.52 \\
\hline 30 & $n-\mathrm{C} 35$ & 3495 & 3500 & & & & & & & $0.18 \pm 0.04$ & 3.95 \\
\hline \multirow[t]{2}{*}{31} & $n-\mathrm{C} 36$ & 3595 & 3600 & & & & & & & $0.08 \pm 0.02$ & 1.66 \\
\hline & Total & & & $81.63 \pm 8.53$ & 99.99 & $68.52 \pm 3.57$ & 100.01 & $65.59 \pm 3.60$ & 100.00 & $3.93 \pm 0.42$ & 100.00 \\
\hline
\end{tabular}

Mean amounts (ng $\pm \mathrm{SE}$ larva $^{-1}$ ) and relative quantities (\% in 1 larval equivalent, LE) are given

${ }^{a}$ Peak numbers refer to Fig. 1a, b

${ }^{\mathrm{b}}$ For the identification procedure see experimental part

${ }^{\mathrm{c}} \mathrm{RI}_{\text {cal }}=$ Retention index calculated on a HP-5MS capillary column ( $\left.30 \mathrm{~m} \times 0.25 \mathrm{~mm} \times 0.25 \mu \mathrm{m}\right)$

${ }^{\mathrm{d}} \mathrm{RI}_{\text {lit }}=$ Retention index as reported for compounds analyzed on HP-5MS or similar columns in the database (http://www.pherobase.com/) and by Fürstenau and Hilker (2017) or others (peak 5 in Hebanowska et al. (1989, 1990) and Svensson et al. (2014); peak 9 in Gerhardt et al. (2016); peak 13 in Spiewok et al. (2006); peak No. 21 in Geiselhardt et al. (2009)). The provided literature RI values of dimethyl alkanes (entries 15 and 17) refer to unambiguously identified compounds described as 5,13-diMeC27 and 3,13-diMeC27

${ }^{\mathrm{e}}$ For the preparation of extracts see experimental part 
Fig. 1 Partial total ion chromatograms (TIC) of a) an unfractionated $O$. surinamensis crude larval extract, b) an unfractionated $T$. confusum crude larval extract (representative for all three Tribolium species as the $\mathrm{CHC}$ profiles were similar in their composition with respect to the presence of $n$-alkanes and methyl alkanes) and c) the fraction of methyl alkanes of fractionated $T$. confusum crude larval extract. Numbers above peaks represent the CHCs listed in Table 2 and Table S5. The $n$-alkanes ( $n$-C25 $n$-C31) detected in the extracts of $T$. confusum and $O$. surinamensis are highlighted in bold. Crossedout compounds are contaminations a)

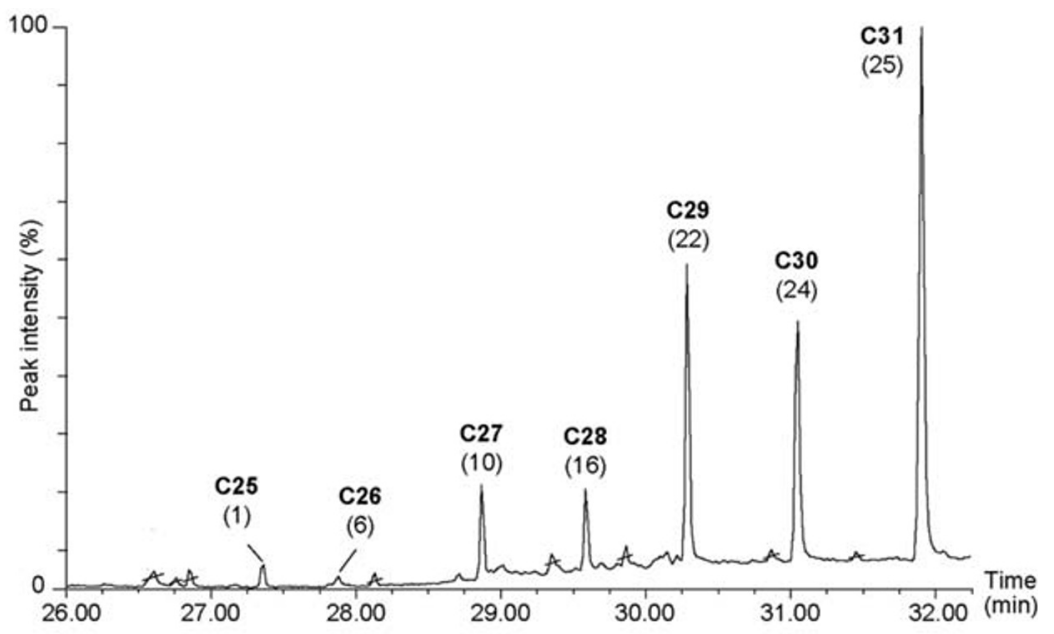

b)

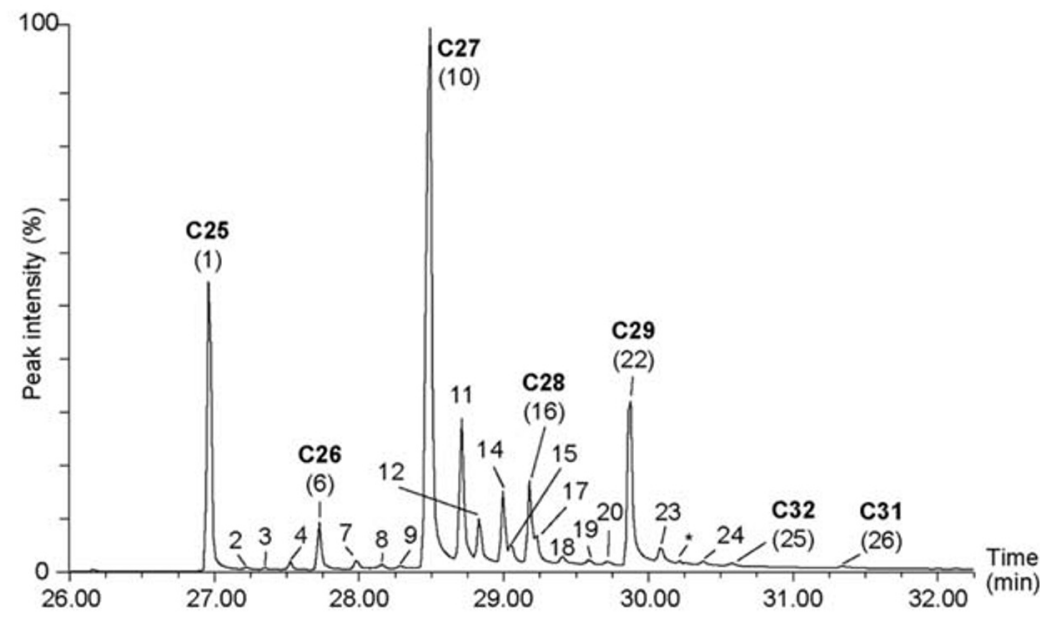

c)

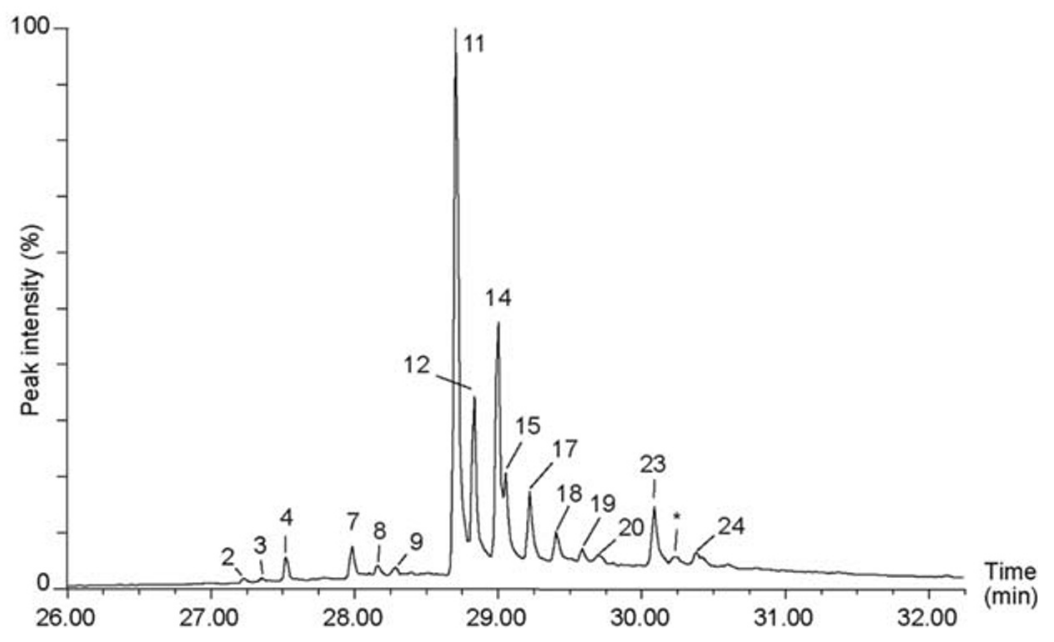

Fractionation of $T$. confusum larval extracts resulted in a clear separation of linear $n$-alkanes on the one hand and monomethyl and dimethyl alkanes on the other (Fig. 1b, c). All methyl alkanes, which had been previously detected in the un-fractionated crude larval extracts, were recovered after fractionation, but in smaller amounts (Table S5). Furthermore, five compounds were detected in the fraction of methyl alkanes (entries 2, 3, 17, 20 and
*), which were not found by our GC-MS analysis of whole T. confusum crude larval extracts (Table 2).

Host Finding and Recognition To test whether the parasitoids' host recognition behavior is mediated by compounds present in the larval CHC profile of all possible host species, we conducted several contact bioassays (Table 1). 


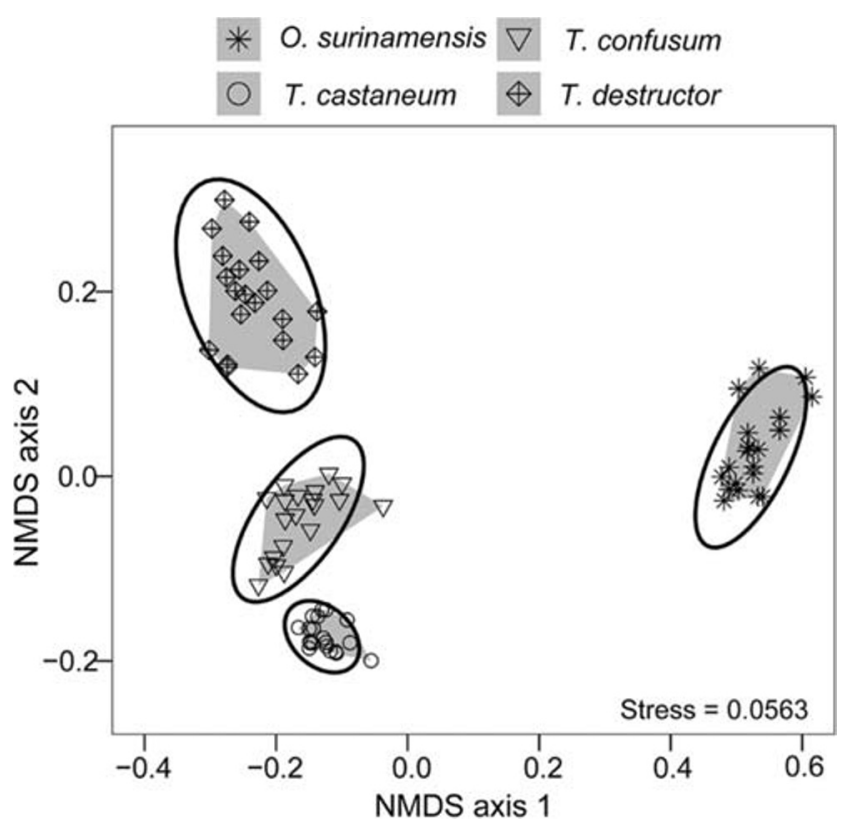

Fig. 2 Comparison of larval CHC profiles of T. castaneum, T. confusum, T. destructor and $O$. surinamensis $(N=20$ per species) visualized by a non-metric multi-dimensional scaling (NMDS) calculated for two dimensions. The ellipses show the $95 \%$-confidence areas around each centroid for each species

When live larvae of different beetle species were offered, $H$. sylvanidis recognized more than $60 \%$ of the T. castaneum and T. confusum larvae as hosts (Fig. 3a). Although the host recognition rate of $T$. destructor larvae was lower (47\%), it did not differ significantly among Tribolium spp., but was significantly higher compared to the rate of $O$. surinamensis larvae $\left(\chi^{2}=37.173, \mathrm{df}=3, P<0.001\right)$. In contrast, the host recogntion rate decreased in all Tribolium spp. when dead larvae were tested (Figure S2a). Nevertheless, the proportion of successfuly recognized host larvae was significantly higher in Tribolium spp. than in O. surinamensis ( $\chi^{2}$ $=30.424, \mathrm{df}=3, P<0.001)$. Live and dead $O$. surinamensis larvae were not recognized as hosts (Fig. 3a, S2a).

Holepyris sylvanidis tended to locate live larvae of T. castaneum (Figs. $3 \mathrm{~b}$ and $80.6 \pm 17.3 \mathrm{~s}$ ) and T. destructor $(79.4 \pm 16.8 \mathrm{~s})$ faster than those of $T$. confusum (102.8 \pm $18.4 \mathrm{~s})$. However, the mean searching times until successful host recognition were not significantly different $\left(\chi^{2}=1.42\right.$, $\mathrm{df}=2, P=0.49)$. When dead larvae were offered, the mean searching time increased in all Tribolium spp. (Figure S2b, T. castaneum $=115.3 \pm 21.3 \mathrm{~s}, T$. confusum $=134.1 \pm 21.1 \mathrm{~s}$, T. destructor $=109.3 \pm 20.7 \mathrm{~s}$ ), but did not differ significantly among them $\left(\chi^{2}=0.66, \mathrm{df}=2, P=0.72\right)$.

Based on these results, we selected $T$. confusum as representative host species for Tribolium spp. and $O$. surinamensis as non-host species for subsequent contact bioassays to investigate the influence of different larval CHC profiles on the host recognition behavior of $H$. sylvanidis (Table 1). Parasitoid females responded significantly different to the four
Influence of beetle species - Live larvae

a)

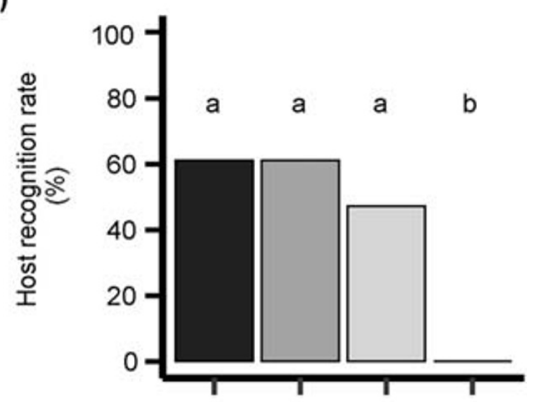

b)

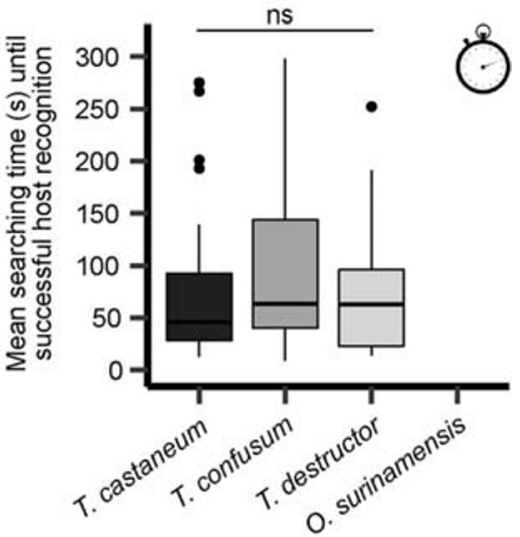

Fig. 3 Behavioral responses of female $H$. sylvanidis in contact bioassays to live larvae of potential host species ( $T$. castaneum, $T$. confusum, T. destructor and $O$. surinamensis; $N=36$ per species, max. observation time $=300$ s). a) Host recognition rate $(100 \% \hat{=} 36$ successful host recognition events per beetle species) was analyzed by the test for equality of proportions followed by pairwise comparison of proportions with Bonferroni-Holm correction. Different letters indicate significant differences at $P<0.05$. b) Mean searching time until successful host recognition was analyzed for Tribolium spp. and not for $O$. surinamensis as larvae of the latter species were rejected as potential hosts for the parasitoid. Statistical analysis was performed by KruskalWallis test (ns $=$ not significant, $P>0.05$ )

treatments of $T$. confusum larvae (Fig. $4 \mathrm{a}, \chi^{2}=19.164, \mathrm{df}=$ $3, P<0.001$; treatments \#5-8 in Table 1 ). While $60 \%$ of untreated larvae were successfully recognized as hosts, larvae became significantly less attractive for the parasitoid, when $\mathrm{CHCs}$ had been removed by solvent extraction (17\%). Applying a $\mathrm{CHC}$ extract of $O$. surinamensis larvae onto previously extracted $T$. confusum larvae, led to a slightly increased amount of successfully recognized host larvae $(20 \%)$. However, this number of successfully recognized larvae did not significantly differ from the one recorded in the previous treatment with extracted larvae. In contrast, the attractiveness of extracted $T$. confusum larvae was restored after applying a CHC extract of $T$. confusum larvae (53\%, Fig. 4a). Furthermore, $O$. surinamensis larvae became only attractive to parasitoid females after extracting their CHCs and applying a larval extract of $T$. confusum (Fig. $4 \mathrm{~b}, P<0.001$; treatments \#9-12 in Table 1). No statistical differences in the mean 
Influence of host larval CHCs

a) $T$. confusum

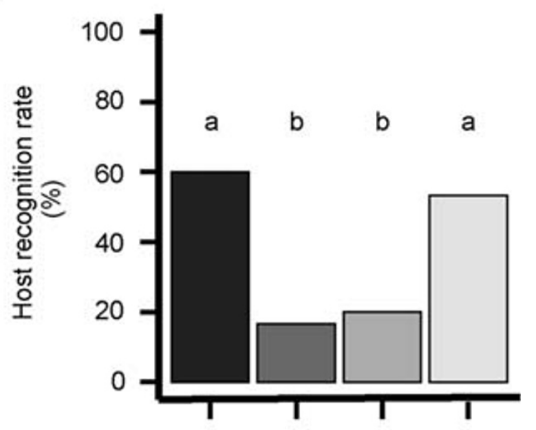

b) O. surinamensis

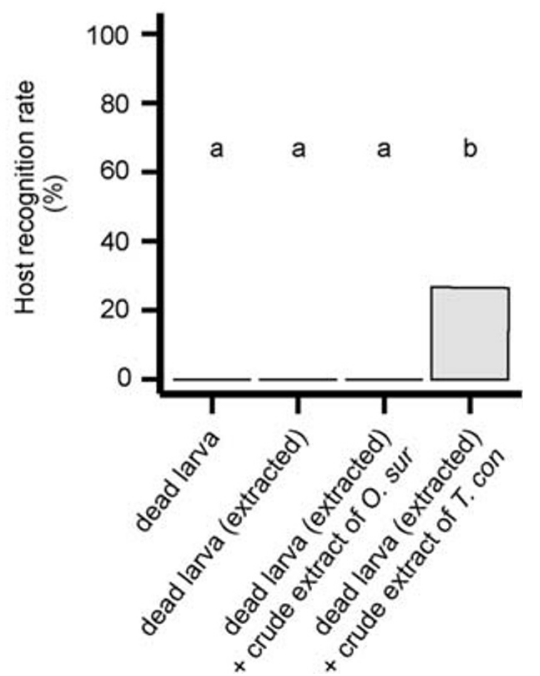

Fig. 4 Host recognition rate in contact bioassays of female $H$. sylvanidis exposed to freshly killed larvae of a) T. confusum (T. con) and b) O. surinamensis $(O$. sur $)$ treated as follows $(N=30$ per species and stimulus, $100 \% \triangleq 30$ successful host recognition events per stimulus, max. observation time $=300 \mathrm{~s}$ ): (i) untreated, (ii) extracted with $n$-hexane, (iii) extracted with $n$-hexane and treated with crude larval extract of $O$. surinamensis ( $1 / 2 \mathrm{LE})$, (iv) extracted with $n$-hexane and treated with crude larval extract of $T$. confusum ( $1 / 2 \mathrm{LE})$. Host recognition rate was analyzed by the test for equality of proportion (T. confusum) or Fisher's exact test (O. surinamensis) followed by pairwise comparison of proportions with Bonferroni-Holm correction. Different letters indicate significant differences at $P<0.05$

searching time of $H$. sylvanidis were found between the different combinations of treatments and larvae (Figure S3, $\chi^{2}=$ 4.836, $\mathrm{df}=4, P=0.305)$.

To elucidate which substance group within the $\mathrm{CHC}$ profile of $T$. confusum might be responsible for host recognition of H. sylvanidis, different $\mathrm{CHC}$ fractions were tested in contact bioassays. Applying the fraction of methyl alkanes onto previously extracted larvae was sufficient to induce host recognition behavior in parasitoid females (Fig. 5a; treatments \#1316 in Table 1). The host recogntion rate $(50 \%)$ was not significantly different from that of untreated larvae $(60 \%)$ as well as of larvae, which had been extracted and treated with whole larval extracts (un-fractionated, 53\%). In these three a)

\section{Influence of host larval $\mathrm{CHC}$ samples}

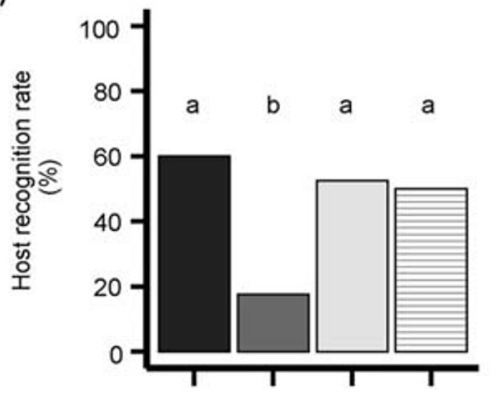

b)

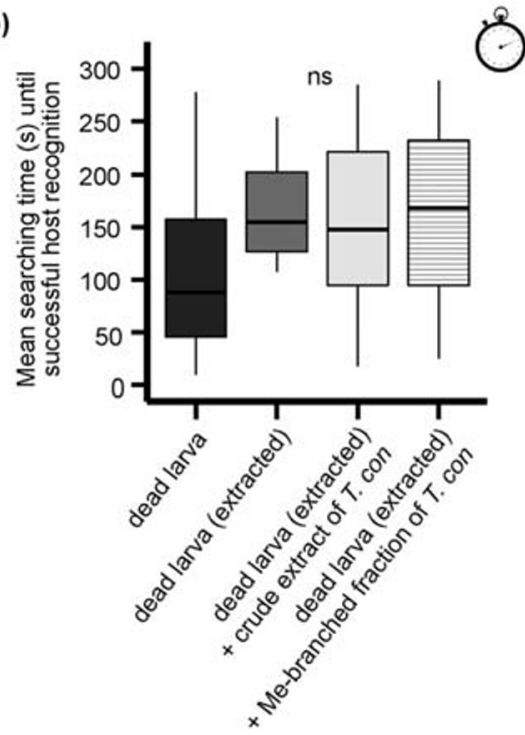

Fig. 5 Behavioral responses of female H. sylvanidis to freshly killed T. confusum larvae treated as follows ( $N=40$ per stimulus): (i) untreated, (ii) extracted with $n$-hexane, (iii) extracted with $n$-hexane and treated with an un-fractionated larval extract of $T$. confusum ( $1 / 2 \mathrm{LE}$ ), (iv) extracted with $n$-hexane and treated with the fraction of methyl alkanes from fractionated $T$. confusum crude larval extract ( $1 / 2 \mathrm{LE})$. a) Host recognition rate $(100 \% \bumpeq 40$ successful host recognition events per stimulus) was analyzed by the test for equality of proportions followed by pairwise comparison of proportions with Bonferroni-Holm correction. Different letters indicate significant differences at $P<0.05$. b) Mean searching time until successful host recognition was analyzed by Kruskal-Wallis test (ns = not significant, $P>0.05$ )

treatments significantly more larvae were successfully recognized as hosts compared to the treatment in which previously extracted larvae were offered $\left(18 \% ; \chi^{2}=17.172, \mathrm{df}=3\right.$, $P<0.001)$. Although $H$. sylvanidis located and recognized untreated $T$. confusum larvae slightly faster than the treated ones, the mean searching time did not differ among treatments \#13-16 (Table $1 ; \chi^{2}=7.196, \mathrm{df}=3, P=0.07$; Fig. 5b).

Host Acceptance Neither live nor freshly killed $O$. surinamensis larvae had been recognized as hosts in contact bioassays, but we included larvae of this beetle species for control purposes (e.g. to test the possibility that the lack of host recognition response might be due to a short test duration $(t=300 \mathrm{~s}))$. 
After exposure of larvae to the parasitoid for $24 \mathrm{~h}$, more than $50 \%$ of the 40 offered larvae of each Tribolium species were parasitized (T. castaneum $=29, T$. confusum $=27$, T. destructor $=23$; Table S6). The parasitoid successfully completed its development from egg to adult on more than $89 \%$ of all parasitized host larvae within four weeks (T. castaneum $=26, T$. confusum $=25, T$. destructor $=22$; Table S6). Most parasitoid adults emerged from parasitized T. castaneum larvae (hatching rate $=65 \%$ ), but no significant differences among Tribolium spp. were observed ( $T$. confusum $=63 \%$, T. destructor $=55 \%$; Figure S4). No parasitoid eggs were laid on $O$. surinamensis larvae within a $24 \mathrm{~h}$ exposure period and thus, host acceptance of this species significantly differed from those of Tribolium spp. $\left(\chi^{2}=45.624\right.$, $\mathrm{df}=3, P<0.001)$.

\section{Discussion}

Our study shows that a larval ectoparasitoid, $H$. sylvanidis, which is supposed to be polyphagous on beetle larvae infesting stored products, selects its hosts by responding to methyl alkanes of the host cuticle. While live and dead 4th instars of the tested Tribolium spp. were successfully recognized as hosts by parasitoid females, larvae of $O$. surinamensis were not, although it had been described as host species in the past (Amante et al. 2017b; Evans 1969; Hagstrum and Subramanyam 2009). Chemical analysis of crude larval extracts revealed that the CHC profiles of Tribolium spp. and those of $O$. surinamensis varied qualitatively and quantitatively. The cuticular extracts of the four investigated beetle species contained $n$-alkanes. The major difference between the CHC patterns was the presence of methyl alkanes, which represented a minor fraction on the cuticle of Tribolium spp., but were absent in $O$. surinamensis. A series of contact bioassays with dead and live larvae of the different beetle species showed that the parasitoid recognizes and accepts a potential host based on the presence of methyl alkanes on the cuticle of an offered larvae. Moreover, a similar response in $H$. sylvanidis was provoked by treating dead, extracted $T$. confusum larvae solely with a fraction of methyl alkanes isolated from $T$. confusum larvae. Therefore, we suggest that host recognition behavior of $H$. sylvanidis is most likely mediated by methyl alkanes from the cuticle of Tribolium spp. larvae, whereas the presence of $n$-alkanes alone is not sufficient to elicit host recognition in the parasitoid.

To the best of our knowledge, our results show for the first time that methyl alkanes are essential to elicit host recognition behavior of a parasitoid species. However, methyl alkanes are well known to elicit behavioral responses in sexual communicaton of parasitoid species belonging to the Encrytidae (Ablard et al. 2012) and Pteromalidae (Kühbandner et al. 2012b; Ruther et al. 2011; Steiner et al.
$2005,2007)$ here the methyl alkanes serve as contact sex pheromones. A recent study by Würf et al. (2020) of a pteromalid species suggests that cuticular methyl alkanes may also act synergistically with $n$-alkanes as contact sex pheromone. For a broad range of non-parasitic insect taxa, methyl alkanes have also been described as contact pheromones, among them ants, beetles, thrips, and psyllids (De Narbonne et al. 2016; Ginzel et al. 2003a; Guédot et al. 2009; Holman et al. 2010; Lacey et al. 2008; Olaniran et al. 2013; Silk et al. 2011; Spikes et al. 2010; Sugeno et al. 2006). For instance, it has been shown that ants use methyl alkanes to discriminate between a mutualistic partner, aphid species, and a non-partner (Sakata et al. 2017).

To study how different larval CHC profiles and isolated methyl alkanes influence host recognition behavior of $H$. sylvanidis, we used solvent extracted $T$. confusum larvae as dummy larvae as (negative) controls. Some of these larvae were still successfully recognized as hosts by the parasitoid in spite of having been extracted leaving no CHCs. This result suggests that not the entire $\mathrm{CHC}$ blend was extracted and small amounts of some cuticular lipids may have been left on the larval cuticle. Hence, we cannot fully exclude that some remaining CHCs (e.g. $n$-alkanes) and/or compounds other than CHCs might also affect the behavior of the parasitoid.

However, we observed that parasitoid females responded to solvent-extracted $T$. confusum larvae treated with isolated methyl alkanes similarly as to dead non-extracted $T$. confusum larvae with a complete blend of CHCs.

These results demonstrate the relevance of methyl alkanes for host recognition behavior of $H$. sylvanidis. So far, we cannot pinpoint yet, which methyl alkanes are most important. Neither do we know whether compounds with higher boiling points than those detected here are relevant for the parasitoids' host foraging behavior. The $\mathrm{CHC}$ profiles of the closely related Tribolium spp. were slightly different. Nevertheless, larvae of each of these beetle species were successfully recognized and accepted as hosts for oviposition by H. sylvanidis, and the parasitoid progenies emerged successfully from most of the parasitized host larvae. These findings suggest that host foraging $H$. sylvanidis females rely on those methyl-branched host CHCs, which the analyzed Tribolium spp. have in common.

We detected 2 externally methyl-branched alkanes (3$\mathrm{MeC} 25$ and 3-MeC27) and 7 internally methyl-branched alkanes (11-/13-MeC25, 11-/13-MeC27 and 12-/13-/14$\mathrm{MeC} 28$ ), which were present in all Tribolium-CHC profiles; among these compounds, $11-/ 13-\mathrm{MeC} 27$ and 3-MeC27 belonged to the most abundant methyl alkanes in the Tribolium-CHC profiles. These common methyl alkanes might be key components eliciting host recognition behavior in H. sylvanidis (entries 2, 4, 11, 14 and 18 in Table 2 and Table S5). This is supported by other studies, which also found behavioral functions for some of these compounds. In particular, 11-/13-MeC27 and 3-MeC27 are known to act as 
chemical cues affecting various behaviors in different insects, for example mate finding, (nest) mate and kin recognition or brood discrimination (Guédot et al. 2009; Kühbandner et al. 2012b; Salazar et al. 2015; Silk et al. 2011; Sugeno et al. 2006). Both compounds were also detected in traces laid by T. confusum larvae, which elicited trail-following behavior in H. sylvanids females (Fürstenau and Hilker 2017). Further studies must be undertaken to investigate, whether single key components are sufficient alone or in combination to elicit host recogntion behavior of $H$. sylvanidis. It will be necessary to carry out similar bioassays with synthetic methyl alkanes to determine those that are essential for host recognition in H. sylvanidis.

Moreover, the chirality of methyl CHCs should be considered since previous studies have shown that enantiomers of a certain compound and its racemate may evoke different behavioral responses in parasitoids (Silk et al. 2011; Würf et al. 2020). For instance, males of the egg parasitoid Ooencyrtus kuvanae were highly attracted to a blend of $5 S-\mathrm{MeC} 27$ and $(5 R, 17 S)$-DiMeC27, whereas they were repelled by a blend of $5 R$-MeC27 and $(5 R, 17 R)$-DiMeC27 (Ablard et al. 2012).

Larvae of $O$. surinamensis have previously been described as potential host of $H$. sylvanidis since both the parasitoid and beetle species have been found at the same storage facilities (e.g. Eliopoulos et al. 2002). However, the chemically mediated parasitoid-host interaction had not been investigated prior to our study, although the $\mathrm{CHC}$ profiles of $O$. surinamensis larvae and adults have been examined (Howard et al. 1995). In this study, a different and more diverse composition of CHCs was described compared to our analyses. Besides several $n$ alkanes with a chain length from $n$-C21 to $n$-C 35 , which represented the predominant group of compounds in the $\mathrm{CHC}$ profiles, the authors also identified few alkenes and even some methyl alkanes, which we did not find in our investigation. Possible explanations for the differences in the $\mathrm{CHC}$ composition of $O$. surinamensis between the study by Howard et al. (1995) and ours may be the origin of test individuals (different lab strains), different rearing conditions (feeding substrate) and the developmental stage. Howard et al. (1995) did not discriminate between larvae and adults in their analyses of the $O$. surinamensis $\mathrm{CHC}$ profiles. Tested insects had been kept on a mix of whole wheat flour enriched with 5\% brewer's yeast and rolled oats. In contrast, we used only beetle larvae for our analysis and provided them with coarsely ground wheat grist.

From previous studies it is known that insect $\mathrm{CHC}$ profiles may vary depending on strain, age, and diet (see references in Otte et al. 2018; Ngumbi et al. 2020). In particular, changes in the insects' diet may lead to shifts in the composition of CHC profiles since the uptake of amino acids and fatty acids is essential for their biosynthesis, especially that of methyl alkanes (Blomquist and Bagnères 2010; Otte et al. 2014). These diet-induced shifts of $\mathrm{CHC}$ compositions can proceed very fast within a short period of one generation or two weeks, thus altering interactions between insects (Geiselhardt et al. 2012; Kühbandner et al. 2012a). Nevertheless, we observed in our contact bioassays that all attempts of $H$. sylvanidis to antennate $O$. surinamensis were aggressively repulsed by the larvae. To exclude this defense behavior, dead larvae were offered as potential hosts to parasitoid females, but they were still not accepted as hosts, most probably due to the lack of methyl alkanes on their cuticle. Therefore, we consider it rather unlikely that $O$. surinamensis is a suitable host for H. sylvanidis.

In summary, the present study demonstrates that methyl alkanes present on the cuticle of Tribolium larvae mediate host recognition in $H$. sylvanidis and enable parasitoid females to differentiate among host and non-host species. Furthermore, the presence of several identical methyl alkanes in all CHC profiles of Tribolium larvae indicates that parasitoid females identify suitable hosts by using these compounds as key substances. Since Tribolium spp. are worldwide pests of various stored products, it is very likely that diet-induced shifts in the composition of CHCs can occur. Future systematic studies need to address, how changes in the diet can affect the presence of methyl alkanes on the cuticle of host larva. If a diet shift results in significant changes in the pattern of methyl alkanes of host larvae, further studies should elucidate, how such changes affect host recognition by $H$. sylvanidis.

Acknowledgements We gratefully acknowledge Christoph Böttcher (Julius Kühn Institute, Institute for Ecological Chemistry, Plant Analysis and Stored Product Protection, Berlin, Germany) for his support in the fractionation of larval extracts. We also thank Heidrun Anders for help and advice in rearing of $T$. confusum and $H$. sylvanidis as well as Raphael Büchner and Rene Grünewald for technical assistance in the analysis of larval extracts. We also thank two anonymous reviewers for their helpful comments on an earlier version of this manuscript.

Funding Open Access funding enabled and organized by Projekt DEAL.

Open Access This article is licensed under a Creative Commons Attribution 4.0 International License, which permits use, sharing, adaptation, distribution and reproduction in any medium or format, as long as you give appropriate credit to the original author(s) and the source, provide a link to the Creative Commons licence, and indicate if changes were made. The images or other third party material in this article are included in the article's Creative Commons licence, unless indicated otherwise in a credit line to the material. If material is not included in the article's Creative Commons licence and your intended use is not permitted by statutory regulation or exceeds the permitted use, you will need to obtain permission directly from the copyright holder. To view a copy of this licence, visit http://creativecommons.org/licenses/by/4.0/.

\section{References}

Ablard K, Gries R, Khaskin G, Schaefer PW, Gries G (2012) Does the stereochemistry of methylated cuticular hydrocarbons contribute to mate recognition in the egg parasitoid wasp Ooencyrtus kuvanae? J 
Chem Ecol 38:1306-1317. https://doi.org/10.1007/s10886-0120189-4

Ahmed KN, Khatun M, Nargis A, Dey NC (1997) Mating, egglaying and host feeding behaviour of Rhabdepyris zeae Waterston (Hymenoptera: Bethylidae) parasitizing Tribolium confusum larvae. Bangladesh J Sci Ind Res 32:633-637

Amante M, Schöller M, Hardy ICW, Russo A (2017a) Reproductive biology of Holepyris sylvanidis (Hymenoptera: Bethylidae). Biol Control 106:1-8. https://doi.org/10.1016/j.biocontrol.2016.12.004

Amante M, Schöller M, Suma P, Russo A (2017b) Bethylids attacking stored-product pests: an overview. Entomol Exp Appl 163:251-264. https://doi.org/10.1111/eea.12587

Amante M, Russo A, Schöller M, Steidle JLM (2018) Olfactory host location and host preference of Holepyris sylvanidis (Brèthes) (Hymenoptera: Bethylidae), a parasitoid of Tribolium confusum Jacquelin du Val and T. castaneum (Herbst) (Coleoptera: Tenebrionidae). J Stored Prod Res 78:105-109. https://doi.org/10. 1016/j.jspr.2018.06.008

Arnaud L, Lognay G, Verscheure M, Leenaers L, Gaspar C, Haubruge E (2002) Is dimethyldecanal a common aggregation pheromone of Tribolium flour beetles? J Chem Ecol 28:523-532. https://doi.org/ 10.1023/A:1014587927784

Baker JE, Sukkestad DR, Woo SM, Nelson DR (1978) Cuticular hydrocarbons of Tribolium castaneum: Effects of the food additive tricalcium phosphate. Insect Biochem 8:159-167. https://doi.org/ 10.1016/0020-1790(78)90068-9

Barron AB (2001) The life and death of Hopkins' host-selection principle. J Insect Behav 14:725-737. https://doi.org/10.1023/A: 1013033332535

Bello JE, McElfresh JS, Millar JG (2015) Isolation and determination of absolute configurations of insect-produced methyl-branched hydrocarbons. Proc Natl Acad Sci USA 112:1077-1082. https://doi.org/ $10.1073 /$ pnas.1417605112

Blomquist GJ, Bagnères AG (2010) Insect hydrocarbons: biology, biochemistry, and chemical ecology. Cambridge University Press, Cambridge

Clarke KR (1993) Non-parametric multivariate analyses of changes in community structure. Aust J Ecol 18:117-143. https://doi.org/10. 1111/j.1442-9993.1993.tb00438.x

Colazza S, Aquila G, De Pasquale C, Peri E, Millar JG (2007) The egg parasitoid Trissolcus basalis uses $n$-nonadecane, a cuticular hydrocarbon from its stink bug host Nezara viridula, to discriminate between female and male hosts. J Chem Ecol 33:1405-1420. https:// doi.org/10.1007/s10886-007-9300-7

Dani FR, Jones GR, Corsi S, Beard R, Pradella D, Turillazzi S (2005) Nestmate recognition cues in the honey bee: Differential importance of cuticular alkanes and alkenes. Chem Senses 30:477-489. https:// doi.org/10.1093/chemse/bji040

De Narbonne MM, Van Zweden JS, Bello JE, Wenseleers T, Millar JG, D'Ettorre P (2016) Biological activity of the enantiomers of 3methylhentriacontane, a queen pheromone of the ant Lasius niger. J Exp Biol 219:1632-1638. https://doi.org/10.1242/jeb.136069

Dexter E, Rollwagen-Bollens G, Bollens SM (2018) The trouble with stress: A flexible method for the evaluation of nonmetric multidimensional scaling. Limnol Oceanogr Methods 16:434-443. https:// doi.org/10.1002/lom3.10257

Eliopoulos P, Athanasiou C, Buchelos C (2002) Occurrence of hymenopterous parasitoids of stored product pests in Greece. IOBC-WPRS Bullettin 25:127-139

Evans HE (1969) A revision of the genus Holepyris in the Americas (Hymenoptera: Bethylidae). Trans Am Entomol Soc 95:181-209

Fürstenau B, Hilker M (2017) Cuticular hydrocarbons of Tribolium confusum larvae mediate trail following and host recognition in the ectoparasitoid Holepyris sylvanidis. J Chem Ecol 43:858-868. https://doi.org/10.1007/s10886-017-0885-1
Fürstenau B, Adler C, Schulz H, Hilker M (2016) Host habitat volatiles enhance the olfactory response of the larval parasitoid Holepyris sylvanidis to specifically host-associated cues. Chem Senses 41: 611-621. https://doi.org/10.1093/chemse/bjw065

Geiselhardt S, Otte T, Hilker M (2009) The role of cuticular hydrocarbons in male mating behavior of the mustard leaf beetle, Phaedon cochleariae (F.). J Chem Ecol 35:1162-1171. https://doi.org/10. 1007/s10886-009-9704-7

Geiselhardt S, Otte T, Hilker M (2012) Looking for a similar partner: Host plants shape mating preferences of herbivorous insects by altering their contact pheromones. Ecol Lett 15:971-977. https://doi. $\operatorname{org} / 10.1111 / \mathrm{j} .1461-0248.2012 .01816 . x$

Gerhardt H, Betz O, Albert K, Lämmerhofer M (2016) Insect adhesion secretions: similarities and dissimilarities in hydrocarbon profiles of tarsi and corresponding tibiae. J Chem Ecol 42:725-738. https://doi. org/10.1007/s10886-016-0718-7

Gibbs AG (1998) Water-proofing properties of cuticular lipids. Am Zool 38:471-482. https://doi.org/10.1093/icb/38.3.471

Ginzel MD, Blomquist GJ, Millar JG, Hanks LM (2003a) Role of contact pheromones in mate recognition of Xylotrechus colonus. J Chem Ecol 29:1-13. https://doi.org/10.1023/A:1022894419521

Ginzel MD, Millar JG, Hanks LM (2003b) Z)-9-Pentacosene - contact sex pheromone of the locust borer, Megacyllene robiniae. Chemoecology 13:135-141. https://doi.org/10.1007/s00049-0030239-Z

Guédot C, Millar JG, Horton DR, Landolt PJ (2009) Identification of a sex attractant pheromone for male winterform pear psylla, Cacopsylla pyricola. J Chem Ecol 35:1437-1447. https://doi.org/ 10.1007/s10886-009-9725-2

Hagstrum DW, Subramanyam B (2009) Stored-product insect resource, 1st. AACC International, Inc., St. Paul Minnesota

Hammer Ø, Harper D, Ryan P (2001) PAST: paleontological statistics software package for education and data analysis. Palaeontol Electron 4:1-9

Hebanowska E, Maliński E, Dubis E, Pihlaja K, Oksman P, Wiinamäki K, Nawrot J, Szafranek J (1989) The composition of cuticular hydrocarbons of the Tribolium destructor. Comp Biochem Physiol Part B Comp Biochem 93:437-442. https://doi.org/10.1016/03050491(89)90104-1

Hebanowska E, Latowska A, Dubis E, Maliński E, Szafranek J, Pihlaja K, Oksman P, Nawrot J (1990) A comparison of cuticular hydrocarbons of larvae and beetles of the Tribolium destructor. Comp Biochem Physiol Part B Comp Biochem 96:815-819. https://doi. org/10.1016/0305-0491(90)90237-n

Hertlein MB, Thompson GD, Subramanyam B, Athanassiou CG (2011) Spinosad: A new natural product for stored grain protection. J Stored Prod Res 47:131-146. https://doi.org/10.1016/j.jspr.2011.01.004

Holman L, Jørgensen CG, Nielsen J, D’Ettorre P (2010) Identification of an ant queen pheromone regulating worker sterility. Proc R Soc B Biol Sci 277:3793-3800. https://doi.org/10.1098/rspb.2010.0984

Howard RW, Blomquist GJ (2005) Ecological, behavioral, and biochemical aspects of insect hydrocarbons. Annu Rev Entomol 50:371393. https://doi.org/10.1146/annurev.ento.50.071803.130359

Howard RW, Howard CD, Colquhoun S (1995) Ontogenetic and environmentally induced changes in cuticular hydrocarbons of Oryzaephilus surinamensis (Coleoptera: Cucujidae). Ann Entomol Soc Am 88:485-495. https://doi.org/10.1093/aesa/88.4.485

Ingleby F (2015) Insect cuticular hydrocarbons as dynamic traits in sexual communication. Insects 6:732-742. https://doi.org/10.3390/ insects6030732

Kühbandner S, Hacker K, Niedermayer S, Steidle JLM, Ruther J (2012a) Composition of cuticular lipids in the pteromalid wasp Lariophagus distinguendus is host dependent. Bull Entomol Res 102:610-617. https://doi.org/10.1017/S000748531200017X

Kühbandner S, Sperling S, Mori K, Ruther J (2012b) Deciphering the signature of cuticular lipids with contact sex pheromone function in 
a parasitic wasp. J Exp Biol 215:2471-2478. https://doi.org/10. 1242/jeb.071217

Lacey ES, Ginzel MD, Millar JG, Hanks LM (2008) 7 methylheptacosane is a major component of the contact sex pheromone of the cerambycid beetle Neoclytus acuminatus acuminatus. Physiol Entomol 33:209-216. https://doi.org/10.1111/j.1365-3032. 2008.00624.x

Lockey KH (1978) Hydrocarbons of adult Tribolium castaneum hbst. and Tribolium confusum duv. (Coleoptera: Tenebrionidae). Comp Biochem Physiol - Part B Biochem 61:401-407. https://doi.org/ 10.1016/0305-0491(78)90145-1

Menzel F, Blaimer BB, Schmitt T (2017) How do cuticular hydrocarbons evolve? Physiological constraints and climatic and biotic selection pressures act on a complex functional trait. Proc R Soc B Biol Sci 284. https://doi.org/10.1098/rspb.2016.1727

Newcombe RG (1998a) Interval estimation for the difference between independent proportions: comparison of eleven methods. Stat Med 17:873-890. https://doi.org/10.1002/(SICI)1097-0258(19980430) 17:8<873::AID-SIM779>3.0.CO;2-I

Newcombe RG (1998b) Two-sided confidence intervals for the single proportion: comparison of seven methods. Stat Med 17:857-872. https://doi.org/10.1002/(SICI)1097-0258(19980430)17:8<857:: AID-SIM777>3.0.CO;2-E

Ngumbi EN, Hanks LM, Suarez AV, Millar JG, Berenbaum MR (2020) Factors associated with variation in cuticular hydrocarbon profiles in the navel orangeworm, Amyelois transitella (Lepidoptera: Pyralidae). J Chem Ecol 46:40-47. https://doi.org/10.1007/ s10886-019-01129-6

Oksanen J et al (2019) vegan: Community Ecology Package (version 2.56). https://cran.r-project.org/package=vegan

Olaniran OA, Sudhakar AVS, Drijfhout FP, Dublon IAN, Hall DR, Hamilton JGC, Kirk WDJ (2013) A male-predominant cuticular hydrocarbon, 7-methyltricosane, is used as a contact pheromone in the Western flower thrips Frankliniella occidentalis. J Chem Ecol 39:559-568. https://doi.org/10.1007/s10886-013-0272-5

Otte T, Hilker M, Geiselhardt S (2014) The effect of dietary fatty acids on the cuticular hydrocarbon phenotype of an herbivorous insect and consequences for mate recognition. J Chem Ecol 41:32-43. https:// doi.org/10.1007/s10886-014-0535-9

Otte T, Hilker M, Geiselhardt S (2018) Phenotypic plasticity of cuticular hydrocarbon profiles in insects. J Chem Ecol 44:235-247. https:// doi.org/10.1007/s10886-018-0934-4

R Core Team (2019) R: A language and environment for statistical computing. R Foundation for Statistical Computing, Vienna

Ruther J, Döring M, Steiner S (2011) Cuticular hydrocarbons as contact sex pheromone in the parasitoid Dibrachys cavus. Entomol Exp Appl 140:59-68. https://doi.org/10.1111/j.1570-7458.2011.01129. $\mathrm{x}$

Rutledge CE, Millar JG, Romero CM, Hanks LM (2009) Identification of an important component of the contact sex pheromone of Callidiellum rufipenne (Coleoptera: Cerambycidae). Environ Entomol 38:1267-1275. https://doi.org/10.1603/022.038.0438

Sakata I, Hayashi M, Nakamuta K (2017) Tetramorium tsushimae ants use methyl branched hydrocarbons of aphids for partner recognition. J Chem Ecol 43:966-970. https://doi.org/10.1007/s10886-0170891-3
Salazar A, Fürstenau B, Quero C, Pérez-Hidalgo N, Carazo P, Font E, Martínez-Torres D (2015) Aggressive mimicry coexists with mutualism in an aphid. Proc Natl Acad Sci USA 112:1101-1106. https:// doi.org/10.1073/pnas.1414061112

Silk PJ, Sweeney J, Wu J, Sopow S, Mayo PD, Magee D (2011) Contact sex pheromones identified for two species of longhorned beetles (Coleoptera: Cerambycidae) Tetropium fuscum and T. cinnamopterum in the subfamily Spondylidinae. Environ Entomol 40:714-726. https://doi.org/10.1603/en10213

Spiewok S, Schmolz E, Ruther J (2006) Mating system of the European hornet Vespa crabro: male seeking strategies and evidence for the involvement of a sex pheromone. J Chem Ecol 32:2777-2788. https://doi.org/10.1007/s10886-006-9162-4

Spikes AE, Paschen MA, Millar JG, Moreira JA, Hamel PB, Schiff NM, Ginzel MD (2010) First contact pheromone identified for a longhorned beetle (Coleoptera: Cerambycidae) in the subfamily Prioninae. J Chem Ecol 36:943-954. https://doi.org/10.1007/ s10886-010-9837-8

Steidle JLM, Van Loon JJA (2003) Dietary specialization and infochemical use in carnivorous arthropods: Testing a concept. Entomol Exp Appl 108:133-148. https://doi.org/10.1046/j.15707458.2003.00080.x

Steiner S, Steidle JLM, Ruther J (2005) Female sex pheromone in immature insect males - a case of pre-emergence chemical mimicry? Behav Ecol Sociobiol 58:111-120. https://doi.org/10.1007/ s00265-005-0930-x

Steiner S, Mumm R, Ruther J (2007) Courtship pheromones in parasitic wasps: Comparison of bioactive and inactive hydrocarbon profiles by multivariate statistical methods. J Chem Ecol 33:825-838. https://doi.org/10.1007/s10886-007-9265-6

Sugeno W, Hori M, Matsuda K (2006) Identification of the contact sex pheromone of Gastrophysa atrocyanea (Coleoptera: Chrysomelidae). Appl Entomol Zool 41:269-276. https://doi.org/ 10.1303/aez.2006.269

Svensson GP, Gündüz EA, Sjöberg N, Hedenström E, Lassance J-M, Wang H-L, Löfstedt C, Anderbrant O (2014) Identification, synthesis, and behavioral activity of 5,11-dimethylpentacosane, a novel sex pheromone component of the greater wax woth, Galleria mellonella (L.). J Chem Ecol 40:387-395. https://doi.org/10.1007/ s10886-014-0410-8

Van den Dool H, Kratz PD (1963) A generalization of the retention index system including linear temperature programmed gas - liquid partition chromatography. J Chromatogr A 11:463-471. https://doi.org/ 10.1016/S0021-9673(01)80947-X

Vassilakos TN, Athanassiou CG, Saglam O, Chloridis AS, Dripps JE (2012) Insecticidal effect of spinetoram against six major stored grain insect species. J Stored Prod Res 51:69-73. https://doi.org/ 10.1016/j.jspr.2012.06.006

Verheggen F, Ryne C, Olsson P-OC, Arnaud L, Lognay G, Högberg HE, Persson D, Haubruge E, Löfstedt C (2007) Electrophysiological and behavioral activity of secondary metabolites in the confused flour beetle, Tribolium confusum. J Chem Ecol 33:525-539. https://doi.org/10.1007/s10886-006-9236-3

Würf J, Pokorny T, Wittbrodt J, Millar JG, Ruther J (2020) Cuticular hydrocarbons as contact sex pheromone in the parasitoid wasp Urolepis rufipes. Front Ecol Evol 8. https://doi.org/10.3389/fevo. 2020.00180 